\title{
Energy efficient three-layer panels and elastic compliance of their middle layer
}

\author{
Stanislav Petrov ${ }^{1}$, and Vadim Alpatov ${ }^{1, *}$ \\ ${ }^{1}$ Samara State Technical University Institute of Architecture and Civil Engineering, 194, \\ Molodogvardeyskaya St., 443001, Samara, Russia
}

\begin{abstract}
Three-layer panels are referred to light weight energy efficient building envelopes. According to current trends, mineral wool from basalt fiber is preferable to be used as panels middle layer. All three-layers of the construction together account for mechanical properties, though these layers taken separately have very different mechanical properties. The work of such a composite design has a number of features that require careful consideration when calculating the panels for strength. Thus, it has not yet been described how squeeze reduction of a relatively soft middle layer affects the load bearing capacity of a panel. When panels are exposed to external loads, their middle layer is squeezed thus changing the characteristics of the panel. This effect is particularly evident in supporting structures. Besides, squeeze reduction of the middle layer changes its elastic-plastic propeties. The purpose of this work is to study the effect of the middle layer of an energy efficient panel squeeze reduction on its load bearing capacity. When solving this task, the authors worked out a methodology which takes into account squeeze reduction of a middle layer and its effect on load bearing capacity of the panel. The researches introduced an algorithm for solving this task and created a tool that allows to easily receive the exact solution. The paper presents this methodology and describes a computer program for calculating three-layer panels with account of changing elastic compliance of a middle layer. The main result of the work is an extended methodology of calculation of the panels and an obtained engineering tool that allows to quickly obtain an extended solution.
\end{abstract}

\section{Introduction}

Three-layer panels with metal sheathing and efficient insulation material as a middle layer (hereinafter the panels) are used as wall envelopes and roof insulation. The use of mineral wool from basalt fiber adds a good thermal quality to the panels, allowing to noticeably reduce energy consumption for heating buildings [1-6]. Application of thin-profiled metal sheets in panels sheathing, separated by their middle layer thickness, allows to effectively use their geometric characteristics under bending loads and to use these panels as load bearing structures. A self-sustainable work of three-layer frameless panel under bending

*Corresponding author: avu75@mail.ru 
loads is possible when additional supporting impact of a hard or semi-hard middle layer (together with its its adhesion with the sheathing) is taken into account [7]. A middle layer on supporting structures is squeezed, its thickness, density, rigidity, elastic compliance, thermal conductivity, etc. changing [8-12]. For a three-layer panel work as a single loadbearing structure, changing in the properties of the middle layer due to its squeeze reduction may become a significant reason for the decline in its load bearing capacity. At present, construction standards and rules do not give a clear answer to the question how to take account of a middle layer squeeze reduction when calculating load bearing capacity of the panel. To simplify the solution of this problem, construction rules suggest using coefficients of structures working conditions for compensation of all negative factors unregistered in calculations, including the middle layer squeeze reduction. However, the rationale for the degree of impact of squeeze reduction of the middle layer is not mentioned among the values of these coefficients, either.

\section{Materials and methods}

The objectives of this work are the study the effect of the middle layer of an energy efficient panel squeeze reduction on its load bearing capacity as well as the introduction of the methodology and the tool to accomplish specified calculations of the panels.

The design of modern energy-efficient panels used in construction, typically consists of two steel sheets, attached to the solid thick middle layer which is more light-weight and less hard. The lower sheet in such panels is generally thin flat or slightly profiled and leans directly on the supporting structure.

The main schemes of these panels work are multi-span continuous schemes. In such conditions, supporting structures flexibility can have a significant impact on the distribution of efforts in construction units.

\section{Results}

The research team conducted experimental studies in the laboratory. The tests were carried out on panels with a length of $4 \mathrm{~m}$, a thickness of $150 \mathrm{~mm}$, working on continuous scheme under evenly distributed load. Deformation of the panel was evaluated by the electric resistive tensometry method with the help of contact deflection indicators and deflection indicators with a wire connection.

It was found that under the load in the sections above the supporting structures, the panel is affected by considerable deformation in its thickness. The amount of deformation of the panels by squeeze reduction is comparable with the value of limit deflections o spans, bearing the whole construction. At the same time, the span of the panel under squeeze reduction in its thickness were not greatly deformed, and in general, work of the construction corresponded to the beam pattern. 

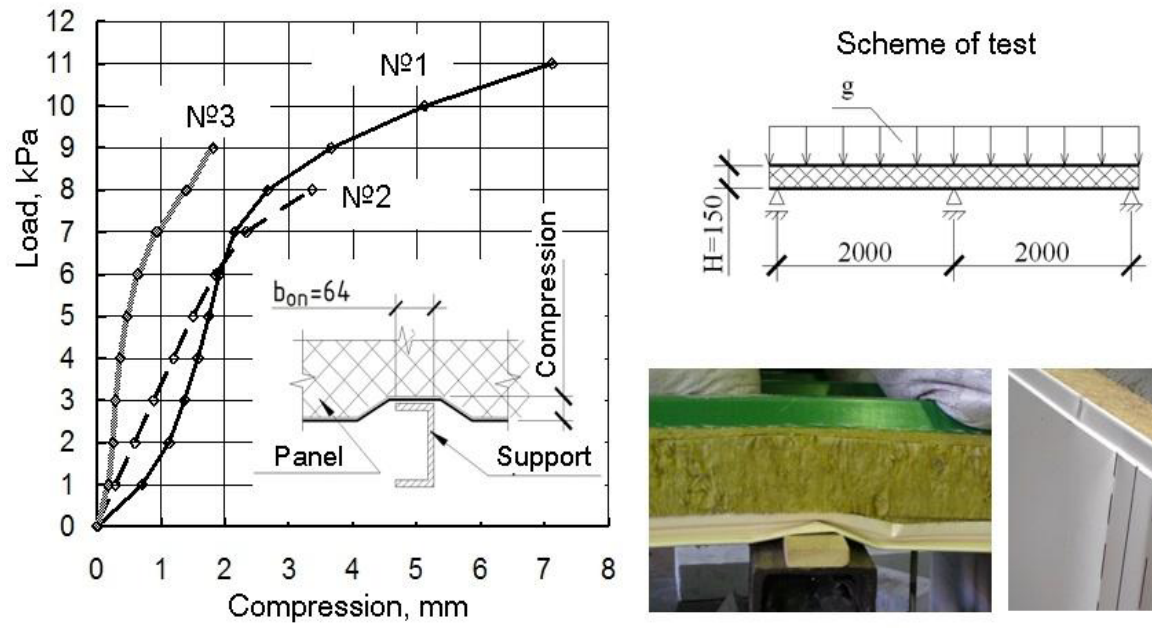

Fig. 1. Design of experiments.
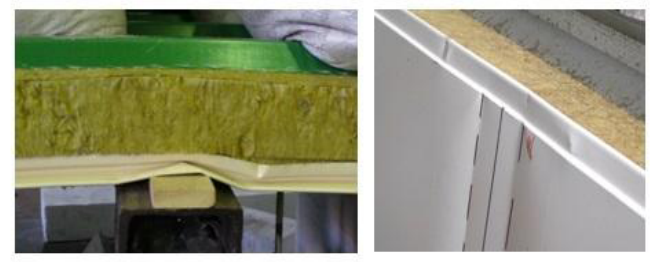

The results of these experimental studies were used to develop a methodology for calculating coefficients of elastic compliance of supporting structures for three-layer panels. The final analytical expression for these calculation is as follows:

$$
k_{\mathrm{i} \text {, up }}=\frac{f_{i \text {,sup }}}{V_{i}}=\frac{5}{384} \cdot \frac{q_{i \text {,sup }} \cdot L_{\text {sup }}^{4}}{E J_{i, \text { sup }}} \cdot \frac{1}{V_{i}}=\frac{5}{384} \cdot \frac{V_{i}}{b} \cdot \frac{L_{\text {sup }}^{4}}{E J_{i, \text { sup }}} \cdot \frac{1}{V_{i}}=\frac{5}{384} \cdot \frac{1}{b} \cdot \frac{L_{\text {sup }}^{4}}{E J_{i, \text { sup }}},
$$

where $f_{i \text { sup }}$ is deflection of $i$ supporting structure corresponding to an appropriate beam; $L_{\text {sup }}$ is a span of the beam; $E J_{\text {isup }}$ is rigidity of a beam section in bending; $q_{i, \text { sup }}$ s the ivalue of beam loads per run.

When solving the problem on the action of the load equal to $q=1 / b_{\text {sup }}$ from support resistance of the panel which is equal to one, we get the following expression to determine coefficients of the panel cross-section flexibility when squeezed on its thickness:

- for midspan supporting structures:

$$
k_{1, d e f}=\frac{1}{E J_{2}} \cdot \frac{1}{\beta^{2} \cdot\left(b_{\text {sup }} \cdot \beta^{2}+4 \cdot \sqrt{0,5 \cdot\left(\alpha^{2}+\beta^{2}\right)}\right)}
$$

- for outer supporting structures:

$$
\left.k_{0, d e f}=\frac{1}{E J_{2}} \cdot \frac{1}{\beta^{2} \cdot\left(2 \cdot b_{\text {sup }} \cdot \beta^{2}+4 \cdot \sqrt{0,5 \cdot\left(\alpha^{2}+\beta^{2}\right.}\right)}\right)
$$

Here $E J_{2}$ is the bending stiffness of the panel lower sheathing; $b_{\text {sup }}$ is the width of the top face of a supporting structure;

$$
\alpha=\sqrt{C_{2} \cdot b / 2 \cdot E J_{2}} ; \beta=\sqrt[4]{C_{1} \cdot b / E J_{2}} ; \mathrm{C}_{1}=E_{3} / H \cdot\left(1-v^{2}\right) \text { is the bedding value of }
$$

elastic foundation under squeezing; $\mathrm{C}_{2}=G_{3} \cdot H / 3$ is the bedding value of elastic foundation under bending; $v$ is the coefficient of transverse deformations of elastic foundation. Other designators are given above. 


\section{Discussion}

Theory of taking into account middle layer elastic compliance when calculating energy efficient three-layer panels. Numerous tests results [13] show that the work of one- and multispan panels can in many cases be analyzed on the basis of beam pattern. Paper [14] introduces the technique of calculating three-layer panels with account of their middle layer shift and elastic compliance of supporting structures on the static effect of evenly distributed shear load $q$. This calculation methodology is based on the model of a composite beam of two bars developed by A.R. Rzhanitsin $[15,16]$. In addition to the above, multispan panels calculation was performed in connection with the force method. Its general view is shown on Figure $3 \mathrm{~b}, \Delta t_{l}, \Delta t_{2}$ is not considered.

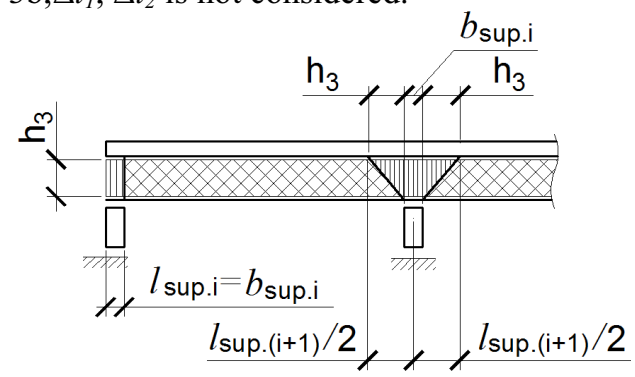

Fig. 2. Determining the size of sites transferring reaction of supporting structures.
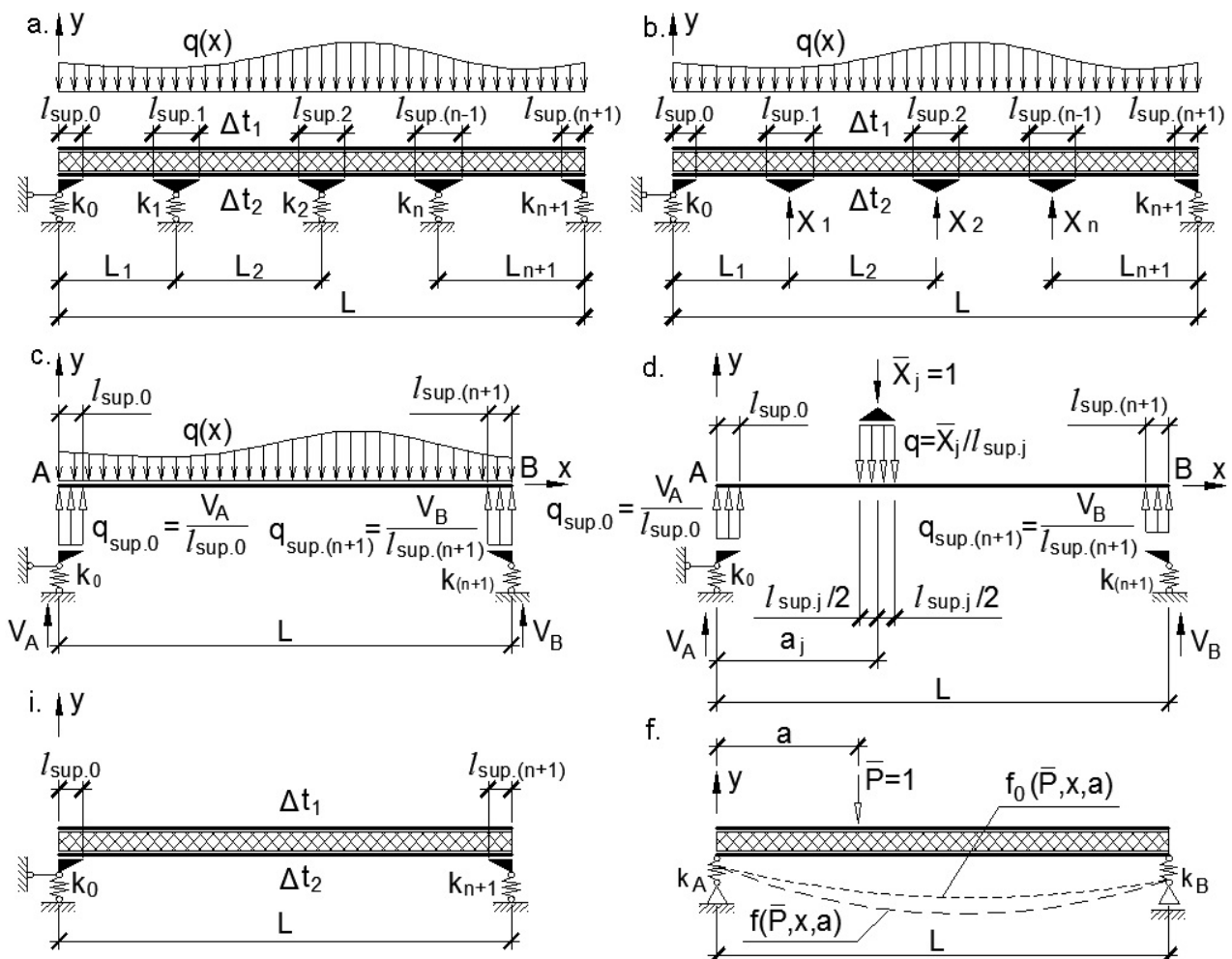

f.

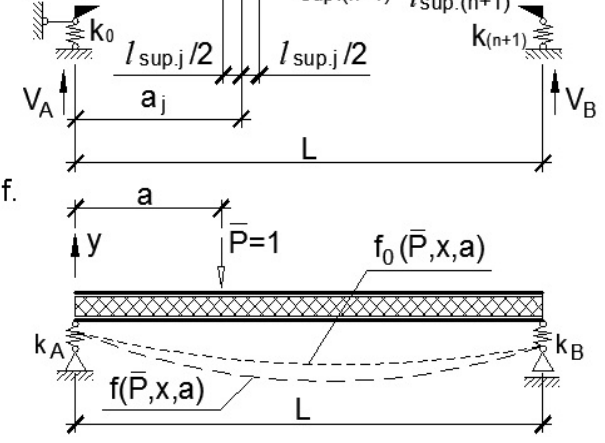

Fig. 3. For determination of the values $\delta_{\mathrm{i}, \mathrm{j}}, \Delta_{\mathrm{i}, \mathrm{p}}$ и $\Delta_{\mathrm{i}, \mathrm{t}}$.

$\mathrm{a}$ - the specified construction design, $\mathrm{b}$ - the force method main system; $\mathrm{c}$ and $\mathrm{d}$ - loaded status of the main system under the action of shear load and thermal effects; $i$ - single state of the main system; $f-$ the basic scheme 
Paper [16] introduces another methodology with two features differentiating features. The first is that the calculation model of the panel considers support resistance to be laid not in the points but distributed through conditional sites of $l_{\text {sup }, i}$ length (see Fig. 2 and Fig. 3a):

$$
l_{\text {sup } . i}= \begin{cases}b_{\text {sup }}+2 \cdot h_{3} & \text { if } \quad 0<i<n, \\ b_{\text {sup }} & \text { if } i=0 \text { and } i=n,\end{cases}
$$

(where $h_{3}$ is the thickness of a middle layer; $b_{\text {sup }}$ is the width of the top sides of the beams; $i$ is the number of supporting structure $(i=0,1,2, \ldots, n) ; n$ being the number of the panel spans). This characteristic is reflected when choosing a single basic system conditions and loaded status of the construction. Figure $3 \mathrm{c}$ and Figure $3 \mathrm{~d}$ demonstrate these schemes. The second feature is that for the determination of displacements and internal forces in cross-sections of single-span three-layer panels, working on the schemes depicted in Fig. 2c and $2 \mathrm{~d}$, we use the expressions obtained for composite beams on elastic supporting structures, loaded by a single concentrated force $P=1$ in Section $a$ (see Fig. 3f).

To develop a general method of calculation of the panels for the joint force impact and the action of temperature differential in sheathings, let's consider the work of a multi-span construction with number of spans corresponding to $n+1$, based on elastic supporting structures with flexibility of $k_{i}$ (see Fig. 3a). Calculation of the panel is done with the force method.

The principal system of the force method is taken according to Figure $3 \mathrm{~b}$. To determine the values of supporting structures reactions in the scheme shown on Figure 3a, let's use a system of canonical equations of the force method proposed in Paper [12], in which the left side of each equation will be supported by a free Member $\Delta_{i, t}$ :

$$
\left.\begin{array}{l}
X_{1} \cdot \delta_{11}+X_{2} \cdot \delta_{12}+\ldots+X_{(\mathrm{n}-1)} \cdot \delta_{1,(\mathrm{n}-1)}+X_{\mathrm{n}} \cdot \delta_{1, \mathrm{n}}+\Delta_{1, \mathrm{p}}+\Delta_{1, \mathrm{t}}=-X_{1} \cdot k_{1}, \\
X_{1} \cdot \delta_{21}+X_{2} \cdot \delta_{22}+\ldots+X_{(\mathrm{n}-1)} \cdot \delta_{2,(\mathrm{n}-1)}+X_{\mathrm{n}} \cdot \delta_{2, \mathrm{n}}+\Delta_{2, \mathrm{p}}+\Delta_{2, \mathrm{t}}=-X_{2} \cdot k_{2}, \\
\ldots \\
X_{1} \cdot \delta_{\mathrm{n}, 1}+X_{2} \cdot \delta_{\mathrm{n}, 2}+\ldots+X_{(\mathrm{n}-1)} \cdot \delta_{\mathrm{n},(\mathrm{n}-1)}+X_{\mathrm{n}} \cdot \delta_{\mathrm{n}, \mathrm{n}}+\Delta_{\mathrm{n}, \mathrm{p}}+\Delta_{\mathrm{n}, \mathrm{t}}=-X_{\mathrm{n}} \cdot k_{\mathrm{n}} .
\end{array}\right\}
$$

where $\delta_{i . j}$ is the displacement along the direction of connections $i$, caused by the action of the load per unit length $q_{\text {sup. } j}=\bar{X}_{\mathrm{j}} / l_{\text {sup. } j}=1 / l_{\text {sup. } j}$ in the single state of the main system of force method (Fig. 3d), attached to replace supporting structures reaction $j ; \Delta_{i, p}$ and $\Delta_{i, t}$ are displacement of the points of an elastic line of the panel over the supporting structure axis $i$ in the loaded state of the main system from the transverse static loads (Fig. $3 \mathrm{c}$ ) and thermal effect (Fig. 3i) respectively; $X_{\mathrm{i}}$ is an unknown resulting force of the supporting structure distributed reaction $i$. On the right side of each equation system (5) there is displacement $i$ of the elastic supporting structure under the effect of the panel reaction $X_{\mathrm{i}}$.

After the solution of the system of equations (5) we find a final equation of elastic line of a multi-span panel exposed to shear load $q$ and temperature difference in sheathing obtained with account of their middle layer shift and elastic compliance of supporting structures . For this purpose it is necessary to sum up the expression of elastic lines of the construction in each $i$ single status (Fig. 3d), multiplied by the value of the corresponding reactions of supporting structures $X_{\mathrm{i}}$, with expressions of elastic lines of the panels in loaded conditions (Fig. 3 c, Fig. 3i):

$$
f(x)=f_{p}(x)+f_{\Delta t}\left(\Delta t_{1}, \Delta t_{2}, x\right)+\sum_{i=1}^{n-1} X_{i} \cdot f\left(x, a_{i}\right)
$$


Here $a_{i}$ the eaxis coordinate of the $i$ supporting structure $(i=1,2,3, \ldots, n-1)$; $f \Delta t\left(\Delta t_{1}, \Delta t_{2, x}\right)$ is determined by taking into account the equality of support reactions to zero; $f_{p}(x)$ is the equation of the elastic line of panels in loaded state from the transverse load, $f_{p}\left(x, a_{i}\right)$ is the equation of the elastic line of a panel in $i$ single state under the action of a single force, distributed at the site $l_{\text {sup. } i}$. The expressions defining functions $f_{\mathrm{p}}(x)$ and $f_{\mathrm{p}}\left(x, a_{i}\right)$ were obtained in Paper [12] by integrating the equation of the elastic line of a beam on a variable, shown in Figure 3c.

\section{Conclusions}

1. Using the correlation (6), according to the methodology [16] that uses a derivation of the equations of elastic line, it is possible to obtain the value of internal stresses, affecting both the entire section of the panel and its separate elements and, therefore, determine the nature of the stress-strain state of a three-layer construction on elastic supporting structures, influencing a static effect of a shear load and temperature differential in its sheathings.

2. Data presented in Paper [16] and obtained by results of a field experiment was compared with the results of calculation of two-span panels according the methodology given in this paper. Both experiments yielded similar results.

3. To automate calculations of the stress-strain state of elements of the three-layer panels by the offered methodology, the researchers developed and algorithm and implemented a program to calculate panels using computer software [17].

The main features of the program include:

- carrying out the calculation of panels with sheathing made of different materials;

- carrying out the calculation of panels with sheathing having different cross-sections;

- carrying out the calculation of panels, working on multi-span schemes with an odd step of supporting structures;

- carrying out the calculation for any combination of static shear strength (in the form of concentrated and distributed loads) and one uniform temperature impact (in the form of temperature difference in panels sheathings);

- taking account of supporting structures flexibility, caused both by bending of the beams and by a local squeeze reduction of a panel on its thickness in the areas above supporting structures (coming from local impacts of supporting structures reactions);

- taking account of physical nonlinear nature of the work of thin lower metal sheathing, when it is under local bending under the influence of supporting structures reactions.

\section{References}

1. Yu.S. Vytchikov, M.E. Saparev, Urban Construction and Architecture, 1(14), 98-102 (2014)

2. Yu.S.Vytchikov, I.G. Belyakov,Proceedings of higher educational institutions. Building, 3, 76-79 (1998)

3. I.V.Kudinov, V.A.Kudinov, E.V.Kotov, etc., Thermophysics of high temperatures,6, 912-92 (2013)

4. A.A. Kudinov,Heat and mass transfer (Moscow, INFRA-M,2014)

5. Yu.S. Vytchikov, M.E. Saparev, Urban Construction and Architecture, 1(22), 4 (2016)

6. Yu.S. Vytchikov, I.G. Belyakov, Urban Construction and Architecture, 4(12), 82-86 (2013)

7. M.M. Kopytov,Metal structures of frame buildings (2016) 
8. E.V.Ildiyarov, I.S.Kholopov, M.D. Mosesov,Modern problems of science and education, 1, 174(2012)

9. E.V. Ildiyarov, Structural Mechanics and calculation of structures, 6, 11-15 (2011)

10. E.V.Ildiyarov, I.S. Kholopov, Industrial and civil engineering, 2, 25-28 (2012)

11. E.V.Ildiyarov, M.D.Mosesov, I.S. Kholopov, The patent of the Russian Federation № 2509997 (2012)

12. E.V.Ildiyarov, I.S. Kholopov,Industrial and civil engineering, 9, 31-36 (2015)

13. S.M.Petrov, E.V.Ildiyarov, N.V.Popkov, etc., Industrial and civil engineering, 5, 44-45 (2009)

14. S.M. Petrov, Structural Mechanics, 4, 27-33 (2010)

15. A.R. Rzhanitsyn, Compound bars and plates (Moscow, Stroiizdat, 1986)

16. A.R. Rzhanitsyn, Structural mechanics. Textbook for high schools (Moscow, Higher school, 1982)

17. S.M. Petrov, Structural Mechanics, 1(246), 23-28 (2013) 